\title{
RESEARCH ON THE INTENSITY ANALYSIS AND RESULT VISUALIZATION OF CONSTRUCTION LAND IN URBAN PLANNING
}

\author{
Jian Cui ${ }^{\text {a, *, Bing Dong }}{ }^{\text {a }}$, Jian $\mathrm{Li}^{\text {a }}$, Lufeng $\mathrm{Li}^{\text {a }}$ \\ ${ }^{a}$ Collage of Survey And Geo-Informatics, Shandong Jianzhu University, China - sdjzu122@126.com
}

Commission IV, WG IV/3

KEY WORDS: Urban Planning, Construction Land, Floor Area Ratio, GIS, Strength Analysis, Visualization

\begin{abstract}
:
As a fundamental work of urban planning, the intensity analysis of construction land involves many repetitive data processing works that are prone to cause errors or data precision loss, and the lack of efficient methods and tools to visualizing the analysis results in current urban planning. In the research a portable tool is developed by using the Model Builder technique embedded in ArcGIS to provide automatic data processing and rapid result visualization for the works. A series of basic modules provided by ArcGIS are linked together to shape a whole data processing chain in the tool. Once the required data is imported, the analysis results and related maps and graphs including the intensity values and zoning map, the skyline analysis map etc. are produced automatically. Finally the tool is installation-free and can be dispatched quickly between planning teams.
\end{abstract}

\section{INTRODCTION}

Urban planning is a complex process that requires collaboration between different disciplines, managing heterogeneous data from different data sources while collecting a large amount of attribute information related to the spatial location of the planning area in order to do processing, analysis and application (Chen Weiqing and Liu Yanhua, 2009). The introduction of GIS (Geographic Information System) in the area of urban planning facilitates the transition of planning from traditional mapping software e.g. CAD to new geographic information platforms e.g. ArcGIS, to help with the convergence of multisectoral planning projects (ZhaiJian and JinXiaochun, 2001). Urban data such as urban status, socioeconomic and geospatial data serves as an important basis for urban planning. Data-based GIS spatial modeling methods can provide good decisionmaking services for traditional urban planning and help planners conduct quantitative analysis when it comes to urban planning issues (Li Miaoyi and Wang Peng, 2014).

In the past two decades, GIS has been widely used in urban planning management in both domestic and foreign projects. Researchers have experimented with the combination of traditional urban residential area location models and GIS to assist in residential planning (Han Sunsheng and Peng Zhen, 2001). In the past, researchers have used Model Builder tools and scripts to create a city park location model that is based on spatial analysis methods from GIS (Li Xiaojun, 2007). The modules i.e. network analysis and spatial analysis embedded in ArcGIS have also been used in combination with Model Builder to study the flood avoidance migration decision model ( $\mathrm{Li}$ Chaojie et al., 2007). Spatial processing modeling techniques has also been employed to establish a suitable location model for skiing (Wang Yu, 2004). Model Builder-based analytic studies have also been conducted on the accessibility of urban catering service industry (Wang Huachen, 2015). Visualization and process modeling functions of Model Builder have also been deployed to evaluate the land suitability of urban planning control in mountainous region, which makes the construction work more scientific and efficient (Li heping and Wang zhuo, 2016). This paper use GIS technology for urban construction intensity analysis on the basis of the above research. When solving practical planning problems, usually, multiple single tools need to be combined in a certain order or placed together in a neat way. We can not complete some of the complex planning and design work by using a single tool. This paper uses GIS technology for urban construction intensity analysis on the basis of the above research. For example, the strength analysis of construction land needs to be calculated by using the panel data of construction land as well as a series of GIS technical methods. The traditional way is inefficient and difficult to guarantee the accuracy and rationality of the intensity zoning of construction land. In addition, the traditional methods lack direct geospatial analysis on result data.

This paper, on the one hand, builds batch computation modeling tool for floor area ratio of construction land. Based on current research results, extension and innovation of Model Builder's application will be achieved. In addition, advanced geoprocessing modeling tools capable of batch automatic floor area ratio computation will be developed. On the other hand, based on the spatial analysis and visualization methods of GIS, result data can be used for graded visualization, sectoral statistics as well as skyline analysis etc. This research solves problems like low efficiency and accuracy, difficult in geospatial analysis and direct visualization associated with processing a large amount of data.

\section{TECHNICAL METHODS FOR BUILDING MODEL TOOLS}

ArcGIS's Model Builder can be used to create, edit, and manage model tools. It is a visualizable programming language for building workflows. Given the fact that in urban planning, the data processing of floor area ratio has a certain procedure often involving panel data. Therefore, it is fairly appropriate to use

\footnotetext{
* Corresponding author

E-mail address: sdjzu122@126.com (J. Cui).
} 
model tools to do the batch automation of it. The model tools used in this paper made use of the advanced modeling technique in Model Builder building a complete working throughout. As is shown in Figure 1.

\subsection{Key Techniques}

This paper studies and builds the model tools that use advanced techniques such as filters, iterators, and inline variables in Model Builder. The filter has a data filtering function that gets the specified feature class data from the input geodatabase by entering a filter keyword. Iterators are the key to automated processing. The term "iteration" refers to the automatic repetition of an operation, often referred to as a loop. The inline variable is used to replace the contents of a variable with the contents of another variable, which ensures the ability of the model tools to batch process the data. By visual programming, we implemented a model tool that enables automatic batch data processing.

\subsection{Process Methodology}

This paper adopts the Model Builder in ArcGIS to study the advanced geoprocessing model tools for batch computation of floor area ratio of urban construction. The model tools are composed of four parts, the first is to pre-process the inputting data, which is followed by the spatial relationship processing of data; the results of the previous data will be put for spatial statistical calculation; finally, the floor area ratio numerical calculation is completed. The details are as follows:

2.2.1 Data Pre-processing: The model tool is designed to use file-based geodatabase as the data input. The first part of the model tools is a pre-processing of the feature classes in the input data by using filters, iterators, and data repair functions. The function of the filter is to filter out the data of the building surface elements in the data file and export it separately as the result data, so that it can be processed separately after the process.

The iterative feature class method is used to iterate over the various types of feature class data in the inputting data. The iterator technique can be used to automate the batch processing procedure by computer. Data repair function is used for the examination and reparation of geometric problem in each element in addition to checking and deleting repetitive elements, which is to ensure the successful operation of modeling tools in the following processing procedure.

2.2.2 Dealing with Spatial Relationships: The spatial data processing section is designed to determine the spatial relationship of each building surface and the land area, to assign the unique attribute values of each site to the building surface to distinguish the belonging of building faces. After determining the spatial reference, the elements are used in cracking and clustering methods to calculate the spatial geometric relationship between inputting data before exporting the result data.

2.2.3 Spatial Data Statistics: The surveying of spatial data of the model tool is achieved through calculating the floor area and aggregating the building area within the land area. First, connect the outputting result data of the previous part to the Add Attribute Field tool to store the next calculation data, then connect to the calculation field tool, obtain the data with the building area, and then collect the statistics according to the land number field. Ultimately, get the land area within each land area.

2.2.4 Calculation of floor area ratio of urban construction land: The last part of the model tool is the calculation of the volume rate of the urban construction land. The data obtained in the third part is linked with the land range data through the attribute linking method to obtain the gross floor area data. The formula to calculate the floor area ratio for the construction strength requirement has been given below in formula (1). Finally, export the data of feature class containing the floor area ratio.

$$
\mathrm{FAR}=\sum S * C / S_{D}
$$

Where $\quad$ FAR $=$ Floor Area Ratio

$\mathrm{S}=$ Base Area of Construction

$\sum \mathrm{S} * \mathrm{C}=$ Gross Floor Area

$\mathrm{S}_{\mathrm{D}}=$ Land-using Area

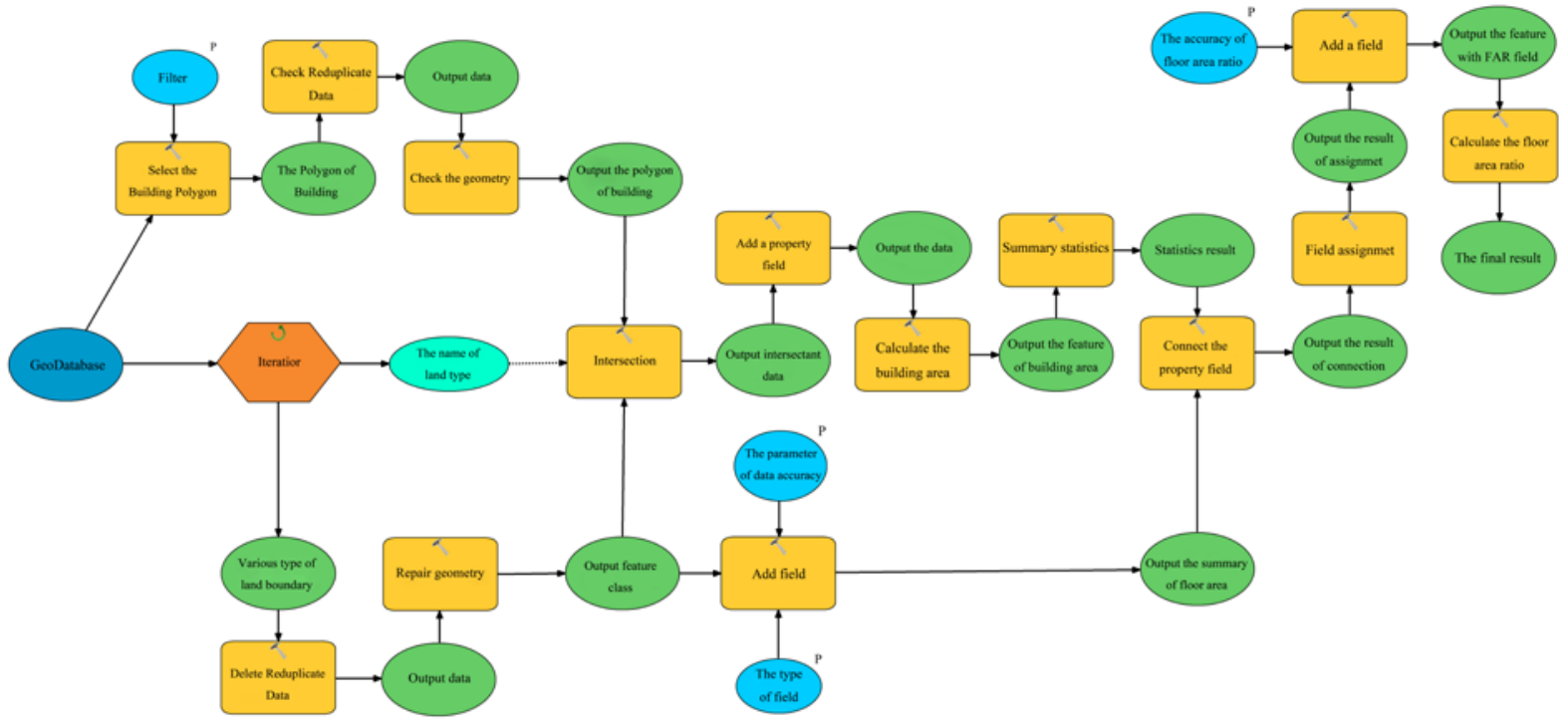

Figure.1 Model tool 

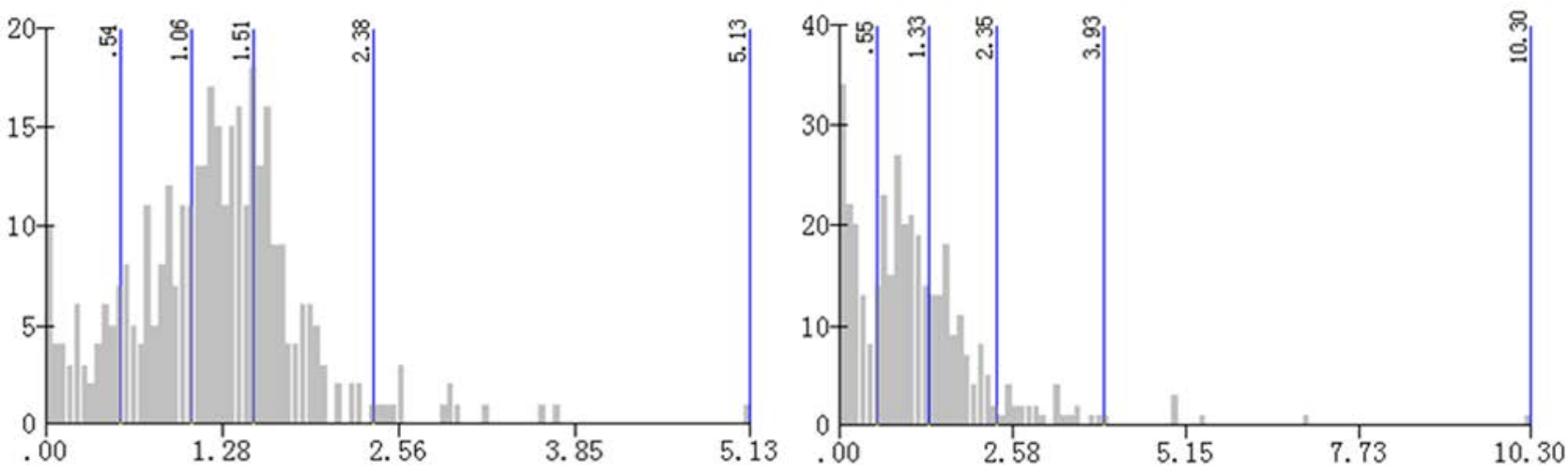

(a)

(b)

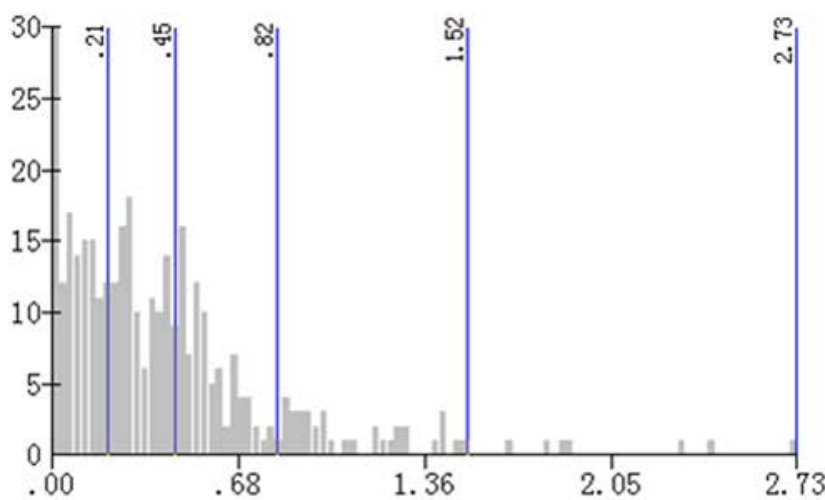

(c)

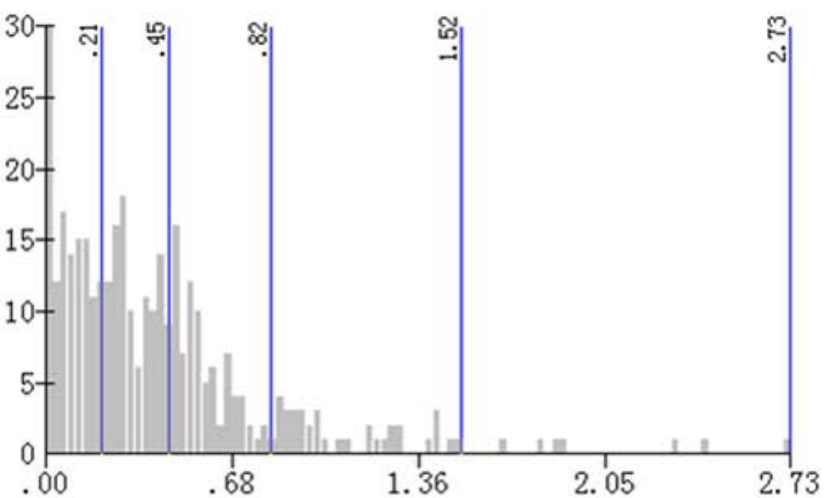

(d)

Figure 2. The classification of floor area ratio of the four types of construction land.

(a) Residential land (b) Commercial land (c) Administrative office land (d) Industry and warehouse land

\section{EXAMPLE OF APPLICATIONS}

This paper uses the research on a city's construction land strength analysis as an instance, in which modeling tool has been applied. The purpose of the study on the strength of urban construction land is to provide the principles and methods of land use intensity control, and provide support data for the determination of land intensity index in planning management, so as to facilitate the implementation of planning management and land use. This paper focuses on the use of spatial data statistics and spatial analysis of GIS technology to carry out the study on the intensity zoning of construction land. This study provides scientific, reasonable and systematic data statistics and data analysis conclusions for urban planning. In the study of urban construction land strength analysis, model tool is used to calculate the volume rate of urban construction land. Then, the result data is used for grading, divisional statistics, spatial visualization and skyline analysis, and the results data is expressed intuitively in the geospatial space, which provides accurate and scientific data support for the analysis of the present situation of the project planning. Finally, the efficiency of the model tool is analyzed.

\subsection{Construction Strength Analysis}

First, import the database of the general construction condition in the research area to model tools. The database of this research area contains four types of land use data and current construction surface feature class data. Data regarding the construction land within the research area will be put for batch automatic calculation. The results reflect the magnitude of numerical value of each construction floor area ratio, but it lacks visibility and spatial distribution. Therefore, the spatial distribution and floor area ratio data of four types of construction land are visualized by using GIS method. Firstly, the Nature Breaks method is used to classify the floor area ratio values, which can identify the classification interval and make the most appropriate grouping of the similar values, so that the differences among the groups can be maximized. According to the actual situation of the area, 5 levels of classification are selected to highlight the differences in the distribution of each type of land in different floor area ratio classification areas. As is shown in Figure 2, the four class classification interval graph to express the construction land intensity in the grading interval (floor area ratio) concentration in number, but only reflect the distribution of various types of construction land intensity, while it lacks geographical space distribution. Then the spatial visualization, as is shown in Figure 3, the expression of different construction strength grading interval by using color depth to the visual display of four different types in the volume of spatial distribution. The four types of construction land data are combined and spatial visualization of all construction land volume values in the study area, as shown in Figure 4. Finally, using spatial statistical methods for statistical construction intensity in order to obtain volume histogram, as is shown in Figure 5, it can be concluded that the average volume of central area was the highest, the West and the high-tech zone is low and very close to each other, southern region, is the lowest, which highlights the difference of construction land distribution in different spatial areas. 

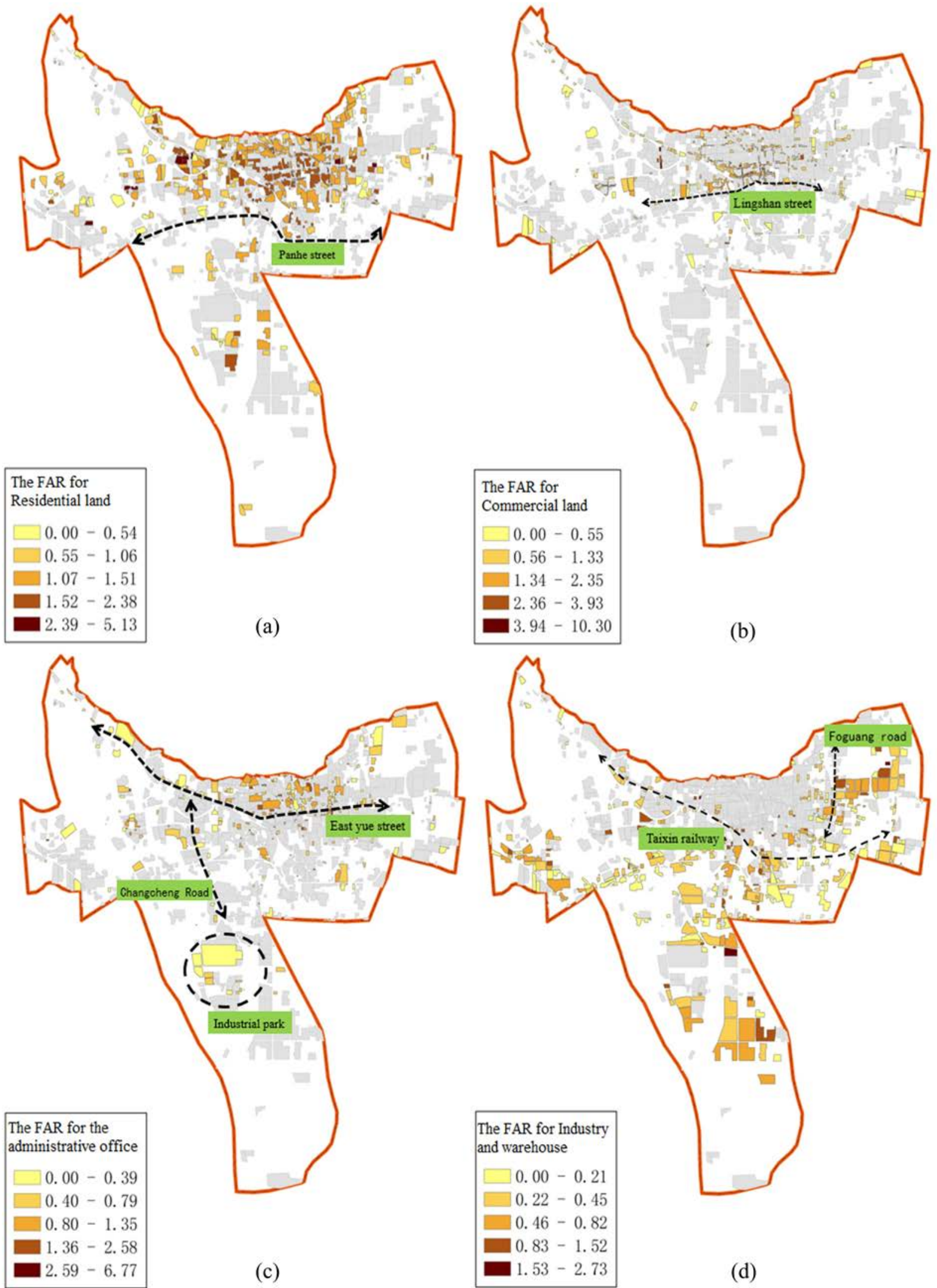

(b)

Figure 3. Spatial visualization of floor area ratio of four types of construction land.

(a) Residential land (b) Commercial land (c) Administrative office land (d) Industry and warehouse land 


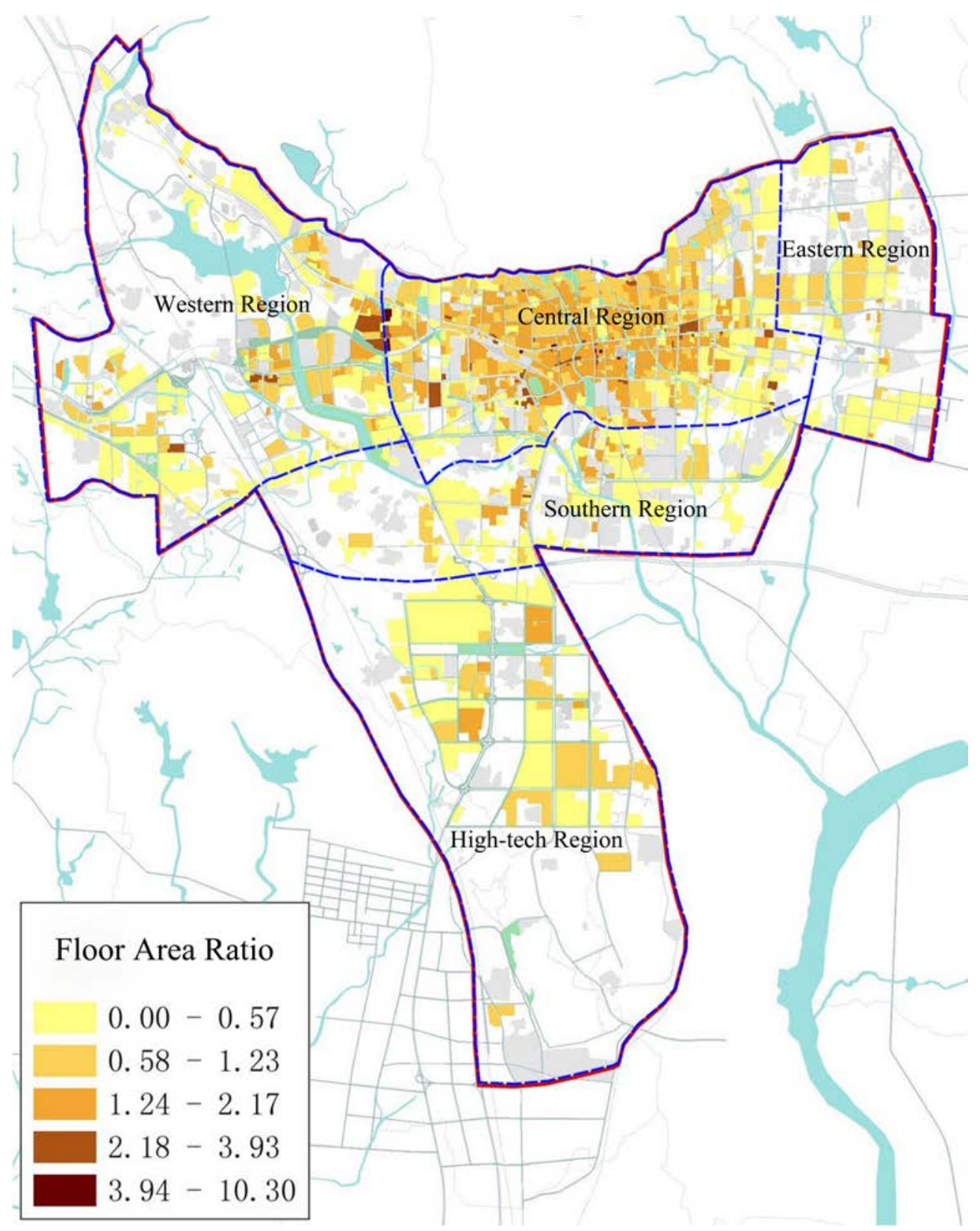

Figure 4. Spatial visualization of floor area ratio of the all construction land

\section{The average of Floor Area Ratio}

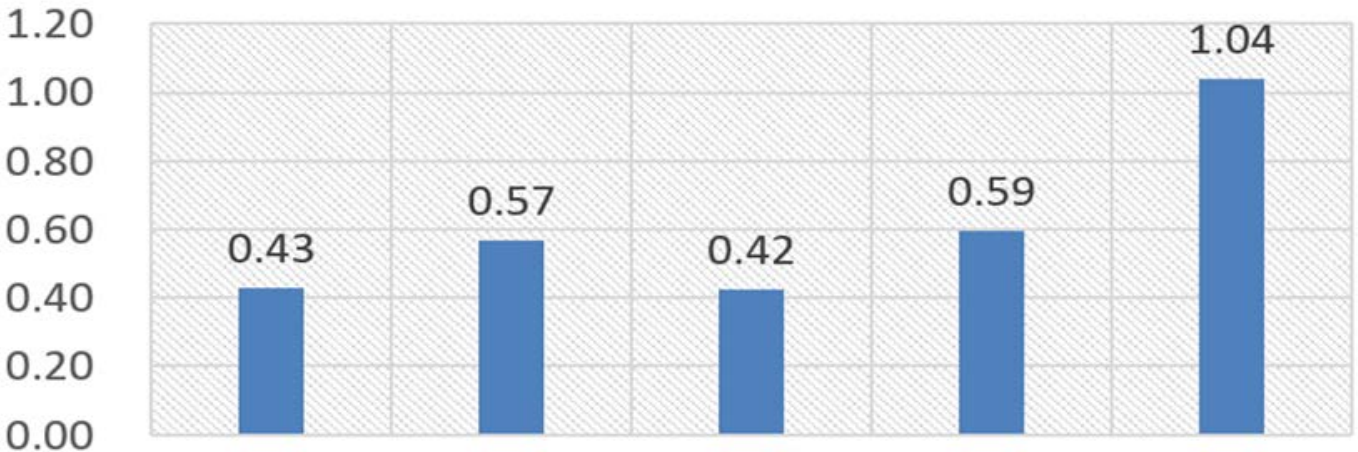

Eastern Region High-tech Region Southern Region Western Region Central Region Figure 5. The divisional statistics of floor area ratio 


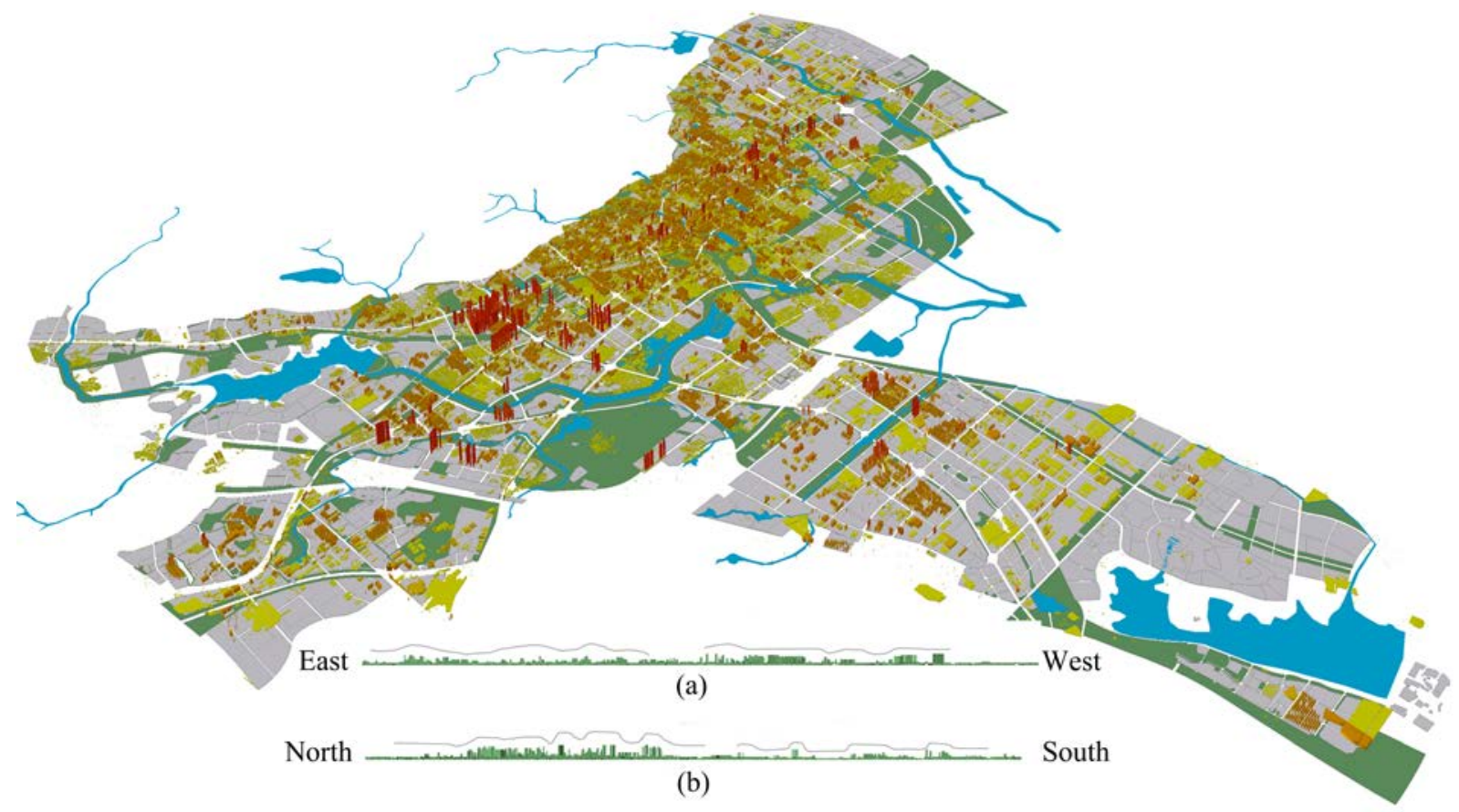

Figure 6. Skyline analysis

(a. The north of skyline, b. The south of skyline)

\subsection{Skyline Analysis}

Using the 3D visualization method of GIS, the threedimensional visualization of the building height in the study area is displayed, and then the data is analyzed by Skyline. As is shown in Figure 6, the northern skyline is observed from the northern to the southern direction. With Wanda high-rise office building as the commanding point, the high-rise buildings appear clustering with the central and western parts have a higher density of high-rise buildings of singular skyline appearance. The eastern part generally has a lower building heights but with richer layer characteristics. The western skyline is observed from the western to eastern direction, the change of skyline in the north of Lai-xin Railway appears to be more diverse. The south part of the railway has a lower building heights of similar characteristics but more consistent.

\subsection{Model Tool Execution Efficiency Analysis}

Taking the strength analysis of a city construction land as an example, the model tool application is applied to the strength analysis of the construction land. About 110,000 building surfaces participated in the calculation, the status of the construction land strength analysis of 1409 construction land participation in the floor area ratio calculation, which also includes the construction land in accordance with the nature of the classification of statistical calculation. The traditional method is to use CAD to conduct the processing of construction land floor area ratio calculation method. It deals with such a large amount of data and takes a long time while can not guarantee the accuracy of the results. With the support of GIS technology, the efficiency has been improved. In the past, ArcGIS was used to calculate the entire building surface data and each kind of construction formation data separately, people proficient in operating this software use such method to calculate construction land floor area ratio, which takes about ten minutes. In this case, there are four types of construction land surfaces, which need to be calculated four times; and the process is a repetitive operation. The above model is used to study the above data, and the efficiency is improved compared with the traditional method. The comparison between methods is shown in Table 7. In addition, the model tool provides a friendly tool parameter interface and can be shared.

\begin{tabular}{|c|c|c|c|}
\hline Type & Size & $\begin{array}{c}\text { Time of } \\
\text { traditional } \\
\text { method }\end{array}$ & $\begin{array}{c}\text { Time of } \\
\text { method used } \\
\text { in this paper }\end{array}$ \\
\hline $\begin{array}{c}\text { Current } \\
\text { data }\end{array}$ & $\begin{array}{c}1409 \text { current } \\
\text { construction land } \\
\text { parcels. 4 types of } \\
\text { construction land } \\
\text { needing } 4 \\
\text { repetitive } \\
\text { calculation. }\end{array}$ & Ca. 0.8 hours & Ca. 3 hours \\
\hline
\end{tabular}

Table 7. Comparison between methods

\section{CONCLUSIONS}

With the extensive application of GIS technology in planning field, planning research has faced a series of changes in methods, which also stimulated the application of GIS technology in urban planning. This paper studies the application of model tool for construction land floor area ratio, data analysis and visualization. Such application can bring the advantage of GIS into the practice of urban planning. Building model tool according to the needs of actual projects decreasing the need for human resources and increasing the processing efficiency of relevant data substantially. The tool is applied to actual project, and its calculation results are visualized and analyzed, and the accuracy is verified. Finally, the efficiency of the implementation of the model tool is compared and analyzed. The results show that the model tool can be used to calculate the volume of floor area in the actual construction. 


\section{REFERENCES}

Han Sunsheng, Peng Zhen, J., 2001. Application of GIS in Foreign Urban Planning. Foreign urban planning,1, pp. 42-44.

Chen Weiqing, Liu Yanhua, J., 2009. City planning multiple source data accordance and database construction; Journal of Guangxi University (Natural Science Edition).1, pp. 106-110.

Li Chaojie, Hu Bin, Gong Huili, Li Xiaojuan, J., 2007. Research and Realization on Decision Model of Evacuation in Flood Disaster, Journal of Capital Normal University (Natural Science Edition). 4, pp. 39-42.

Li Heping, Wang Zhuo. J., 2016. Application of the Land Suitable Evaluation Based on Model Builder in Mountainous Cities Regulatory Detailed Planning-A Case Study of Regulatory Detailed Planning in Jiangdong District, Wushan County, Chongqing Municipality, Journal of Human Settlements in West China.21, pp. 13-18.

Li Miaoyi, Wang Peng, J., 2014. New Technology in Datadriven Urban Planning: From GIS to Big Data. Urban Planning International, 6, pp.58-65.

Li Xiaojun, J., 2007.Study of GIS-Based Spatial Analysis Methods, Zhejiang University. 1, pp.42-44.

Song Xiaodong, NiuXinyi, J., 2010. History and trends of GIS Application in Urban Planning: A Sino-us comparison and prospect, Planning Studies.10, pp. 23-29.

Wang Huachen, J., 2015. Modeling Analysis of Accessibility in City Catering Industry Based on 2SFCA and Model Builder, Geomatics \&Spatial Information Techonology.8, pp. 126-128.

Wang Yu, G., 2004. Establishment of Suitability Location Model, ESRI China (Beijing) Training Center, 2004 training materials.

ZhaiJian, JinXiaochun, J., 2014.Method for GIS spatial analysis in urban planning. City Planning Review.38, pp. 130-13. 